\title{
Zur Situation der erdölexportierenden Länder des Nahen Ostens
}

\author{
Ahmad Tahmassebi
}

Die Ursachen der gegenwärtigen ölkrise lassen sich weder mit der simplen Darstellung von den ,erpresserischen Ölscheichs" ${ }^{66}$ noch mit dem Klischee einer endich im Kampf gegen den Imperialismus geeinten arabischen Welt, ausreichend erklären.

Je weiter man die Entwicklung verfolgte, um so deutlicher zeigte sich, daß nicht die arabisschen ölscheichs, sondern die multinationalen Ölgesellschaften die ausschlaggebenden Kräfte in dieser Krise sind. Eine Analyse wird deshalb um so komplizierter, da diese mächtigen Konzerne weder von den kapitalistischen Regierungen noch von der öffentlichkeit gezwungen werden können, ihre Geschäftsprak. tiken offen zu legen. Die bürgerliche Presse berichtet zwar über all Ereignisse und veröffentlicht alle ihr zur Verfügung stehenden wichtigen Fakten, vermeidet es aber geschickt, diese in den Zusammenhang zu bringen, in den sie gehören und betreibt so eine allgemeine Verwirrung und oft genug eine bewufite Manipulation.

Um aber die Zusammenhänge der Ölkrise verstehen zu können, muß man sich in einer Untersuchung mit den drei wichtigsten Faktoren auseinandersetzen, die die gegenwärtige Situation bestimmen. Das sind einmal die arabischen Länder und insbesondere die ölfördernden Staaten in dieser Region, die sich aufgrund ihrer wirtschaftlichen und politischen Lage für eine Öldrosselung entschieden haben. Zum anderen sind es die Mineralölgesellschaften, die diesen Umstand zum willkommenen Anlaß nahmen, ihre eigenen Interessen besser durchsetzen zu können. Und drittens spielt die sich allgemein abzeichnende Wirtschaftskrise der kapitalistischen Länder eine Rolle, zu deren Verschleierung die sog. Energiekrise beitragen soll. In diesen Ausführungen werden wir uns auf die ersten beiden Faktoren konzentrieren, die komplizierten Zusammenhänge zwischen der ölkrise einerseits und der aligemeinen Wirtschaftskrise in den kapitalistischen Ländern andererseits müssen einer anderen Untersuchung vorbehalten bleiben.

Um die Gründe zu verstehen, die die arabischen ölexportierenden Staaten dazu veranlaßten, einen Ölboykott auszusprechen, wollen wir zuerst einmal kurz auf die Entwicklung des antiimperialistischen Kampfes der arabischen Länder eingehen.

Kurzer Ůberblick über den antiimperialistischen Kampf der arabischen Länder nach. dem II. Weltkrieg

Nach dem II. Weltkrieg nahmen die Befreiungsbewegungen in der Dritten Welt einen starken Aufschwung. Die wenigsten dieser Bewegungen standen unter der Führung einer starken kommunistischen Partei. Die meisten hatten mehr nationalen Charakter und blieben entweder erfolglos oder stagnierten in einer gewissen Phase.

Um eine ähnliche Entwicklung handelte es sich auch in den meisten arabischen Ländern. Da es keine eigenständig gewachsenen kommunistischen Parteien 
gab, standen die sich bildenden sozialistischen Parteien unter dem traditionellen Einfluß der Sowjetunion. Sie waren mehr das Produkt intemationaler Auseinandersetzungen, als das Produkt langwieriger Klassenkämpfe in ihren eigenen Ländern und es gelang ihnen nicht, die Führung der unterdrückten Klassen zu übernehmen. Andererseits war die nationale Bourgeoisie noch zu schwach entwickelt, als daß sie die Bewegungen in ihrem Sinne hätte leiten können.

In dieser Situation gewann die Kleinbourgeoisie in den wichtigsten arabischen Staaten an entscheidendem Einfluß und setzte sich an die Spitze des Kampfes gegen den Imperialismus. Die Kleinbourgeoisie stand zwar im Widerspruch zu den kapitalistischen Industrieländern, es fehlte ihr aber sowohl die ökonomische Grundlage, als auch die revolutionäre Massenbasis, auf der grundlegende Veränderungen in diesen Ländern hätten erfolgreich durchgeführt werden können. Wo eine langfristige Mobilisierung der Massen - wie z. B. im Kampf Algeriens gegen die Kolonialherrschaft Frankreichs - nicht möglich war, versuchte die Kleinbourgeoisie in der Armee Fuß zu fassen, um mit einem Putsch die politische Macht zu übernehmen und somit die Möglichkeit für ihre Entfaltung zu schaffen. Diese Form der Machtïbernahme finden wir auch in den meisten arabischen Ländern und insbesondere in Ägypten.

In Agypten ist die Phase von 1952-56, d. h. zwischen der Machtübernahme der Offiziere und der direkten Intervention englischer, französischer und israelischer Truppen, die Zeit, in der Nasser und seine Offiziere als Vertreter der Kleinbourgeoisie ihre Position festigen konnten. Es war für sie unbedingt wichtig, für ihre Auseinandersetzung mit dem Imperialismus die Massen für sich zu gewinnen. Der politische Erfolg während der Suezkrise brachte Nasser die volle Unterstützung der antiimperialistischen Massen.

Die Sowjetunion, von deren Politik und der ihrer Bruderparteien (besonders wegen der Ereignisse nach dem II. Weltkrieg im. Zusammenhang mit der Gründung des Staates Israel) die arabische Bevölkerung bislang enttäuscht gewesen war, konnte mit ihrer massiven Unterstützung der Nasserregierung ihr Ansehen in diesen Ländern beträchtlich steigern. Dort zeigten sich zwar auch jetzt keine starken Sympathien für eine marxistische Bewegung, aber man hatte auch keine traditionelle Abneigung gegen die sozialistische Gesellschaftsordnung.

Die Phase von 1956 bis Anfang der 60er Jahre brachte der Nasserregierung permanente politische Erfolge und die allmähliche ökonomische Entwicklung der Kleinbourgeoisie. Da es am Anfang wegen der starken Unterentwicklung in fast ailen Bereichen kaum möglich war, privates Kapital in die Industrie zu ziehen, mußste der Staat die Industrialisierung des Landes mit öffentlichen Mitteln finanzieren. Diese staatlichen Aktivitäten ermöglichten es der Bourgeoisie und der Kleinbourgeoisie sich leichter auf dem Gebiet des Handels und der Industrie zu engagieren. Außerdem konnten sich die - durch die Bodenreform entmachteten, aber materiell entschädigten - Großgrundbesitzer an der Kapitalisierung des Landes beteiligen. Schließlich bildete sich noch eine bürokratische Schicht im öffentlichen Sektor, die auch stark im privaten Wirtschaftsbereich Kapital investierte.

All diese Faktoren schufen Anfang bis Mitte der 60er Jahre eine Situation, in der die wachsende einheimische Bourgeoisie nach und nach eine starke Position im Staatsapparat eroberte. Parallel zu den innenpolitischen Fortschritten gelangen Nas- 
ser auch Erfolge auf internationaler Ebene und bald wurde er einer der wichtigsten Führerfiguren der Dritten Welt. Sein Einfluß in den arabischen Ländem war so groß, daß sich mit Ausnahme der Volksrepublik Jemen alle Emanzipationsbewegun" gen nach dem ägyptischen Modell richteten.

Bei der Entwicklung der arabischen Kleinbourgeoisie sind vor allem zwei dialektisch-widersprüchliche Faktoren zu berücksichtigen. Einmal ist da die historische antiimperialistische Aufgabe, die sie zu lösen übernommen hat, aber zum ande. ren baute sie natugemäß eine starke Schranke gegen die Emanzipation der Arbeiter- und Bauemklasse auf und verhinderte eine breite Entfaltung des Klassenbewußsteins. Um aber diese Klassen ganz davon zu überzeugen, daß die politische und wirtschaftliche Entwicklung auch in ihrem Interesse gefuhrt wurde, brauchte die Kleinbourgeoisie eine einleuchtende ideologische und theoretische Formulierung, mit der sich alle antagonistischen Widerspriiche äberbricken ließen. Dieser Anspruch wurde mit der Formel vom, ,islamischen Sozialismus" erfillt.

Nassers politische Erfolge waren so groß, daß die arabischen Völker und auch die Palästinenser in ihm den Deus ex machina sahen, der das israelische Problem allein zu lösen vermag. Die Niederlage Ägyptens, Syriens und Jordaniens im JuniKrieg 1967 zerstörte nicht nur diese Illusion, sondern brachte auch viele neue Probleme mit sich:

1. Der Verlust großer arabischer Gebiete und die Schließung des Suezkanals.

2. Verstärkte wirtschaftliche und politische Abhängigkeit von der Sowjetunion.

3. Die arabischen Massen sind von ihrer Führung enttäuscht und die Palästinenser sehen ein, daß sie sich für ihre Interessen selbst mobilisieren müssen. Es bilden sich unabhängige marxistische Zellen, doch im großen und ganzen wird auch diese Bewegung von der radikalen Kleinbourgeoisie beherrscht.

4. Die Bourgeoisie, die speziell in Ägypten in einer starken Verbindung zu der Sowjetunion keine Möglichkeit für ihre Kapitalentwicklung sah, zeigte großes Interesse, sich dem westlichen Kapitalmarkt anzuschließen. Sie geriet in einen scharfen Widerspruch zum radikalen Flügel der Regienng.

\section{Die Entwicklung nach dem Juni-Krieg 1967}

Nasser starb 1970 und überließ die Lösung dieser schwierigen Probleme seinen Nachfolgern.

Sadat, als Vertreter des rechten Flügels des ,Islamischen Sozialismus ${ }^{66}$, übernimmt Nassers Platz. Sabri und die Anhänger des linken Flügels werden als erste ausgeschaltet.

Sadat war von Anfang an bestrebt, die Beziehung zu den USA zu normalisieren. Dies lag im allgemeinen Interesse des expansionsbediurftigen Industrie- und Handelskapitals. Er hoffte auch durch geschicktes diplomatisches Taktieren, die von Israel besetzten Gebiete zurückzuerhalten. Die USA waren auch tatsächllich zu größeren Zugeständnissen bereit und unterbreiteten den Rogers-Plan, der alle Parteien zufriedenstellen sollte. Dieser Plan sah $u$. a. vor, daß Israel einen Teil der besetzten Gebiete räumt, daß die Palästinenser ein Stïck Land erhalten und auf alle 
weiteren Forderungen verzichten sollten. Der Plan - der auch die Unterstützung der Sowjetunion fand - scheiterte aber vor allem aus zwei Gründen. Einmal an der Ablehnung Israels, weiches bis jetzt nur militärische Erfolge kannte und auf keinen seiner Ansprüche verzichten wollte, und zum anderen scheiterte er an dem Widerstand der Palästinenser, die in seiner Durchfihrung ihre totale Kapitulation sahen.

Unter diesen Umständen gab es für Sadat keine andere Möglichkeit, als entweder die Besetzung der arabischen Gebiete zu dulden -- wobei er mit einem Putschversuch der unzufriedenen radikalen Offiziere rechnen mußte - oder die politischen und wirtschaftlichen Erfolge der Nasserregienung zu wiederholen. Für die Durchführurg seiner Pläne brauchte er aber einen starken Verbündeten, der ihn sowohl finanziell als auch ideologisch stark unterstïtzen konnte.

Da er sich noch immer als Vertreter der radikalen Kleinbourgeoisie präsentieren wollte, suchte er zunächst die Unterstïtzung Libyens, dessen Kapitalïberschuß den Plänen der ägyptischen Bourgeoisie sehr nützlich sein könnte. Auch Ghaddafi seinerseits neigte zu einer ägyptisch-libyschen Föderation, allerdings mit einer anderen Motivation. Er ist tatsächlich der Vertreter der radikalen antiimperialistischen Kleinbourgeoisie und er versprach sich aus einer Verbindung mit dem volkreichsten arabischen Nachbarn diese Kleinbourgeoisie zum Herrscher des gesamten arabischen Raumes zu machen - wie es Nasser immer wollte und anfangs auch versuchte (z. B. mit der gescheiterten ägyptisch-syrischen Föderation).

Aber wie konnten die Vorstellungen Ghaddafis, dem Vertreter der verspäteten libyschen Kleinbourgeoisie, in Erfuillung gehen, wenn es selbst Nasser in seiner besonderen historischen Situation nicht gelingen konnte. Er versuchte es trotzdem, aber die verwöhnte ägyptische Bourgeoisie war nicht bereit, seinen Radikalismus zu dulden. Sie wollte das libysche Kapital nach ihrer Vorstellung verwenden und nicht davon kontrolliert werden. Der Marsch von 50000 Libyern, die zur symbolischen Vereinigung beider Staaten an die ägyptische Grenze zogen, fand zwar starke Sympathien bei den Studenten und radikalen Offizieren, aber die ägyptische Bourgeoisie und Staatsbürokratie stoppte den Marsch der Libyer und zerbrach damit alle Wünsche und Illusionen Ghaddafis. Er kritisierte die ägyptische Staatsbürokratie vergeblich, denn Sadat ließ sich davon nicht erschüttern und entschied sich für einen Weg, den er bis jetzt noch nicht so offen zu gehen gewagt hatte. Kurz nach seiner Auseinandersetzung mit Libyen erfuhr die erstaunte Weltöffentlichkeit, daß sich Sadat zu geheimen Gesprächen in Saudi-A rabien aufhielt.

Die öffentlichkeit wunderte sich mit Recht, denn bisher gab es wenig Gemeinsamkeit zwischen dem reaktionären saudiarabischen Regime und der Regierung in Kairo. Agyptische Soldaten kämpften jahrelang in Jemen gegen saudiarabische Einheiten, die die nordjemenitische Regierung unterstïtzten. Saudi-Arabien war auch ein Geldgeber Hosseins, der zweimal auf den Befehl des Pentagons die palästinensischen Fedajin abschlachten ließ. Man mußte vermuten, daß die Gespräche zwischen Sadat und Feisal zu einer Wende in der gesamten arabischen Politik führen würden.

Die Isolation Ghaddafis, die Depression der arabischen Bevölkerung durch die Verluste im Juni-Krieg und die ständigen Niederlagen der palästinensischen Organisationen gaben nun den rechten Kräften die Möglichkeit, ihren Einfluß zu verstär- 
ken, um die arabische Politik in Zukunft stärker nach ihren Vorstellungen zu bestimmen. Die allgemeine Depression wurde auch noch durch die fortwährenden Anschläge der Israelis vertieft. Sie entführten ein Flugzeug, schossen eine libysche Passagiermaschine $a b$, sandten Kommandounternehmen in die palästinensischen Wohnviertel im Libanon, wo sie die Bewohner im Schlaf ermordeten und bombardierten schließlich permanent libanesisches Gebiet.

Die einzige Reaktion auf diese Aggressionen war entweder das Protestgeschrei Ghaddafis, der sich nach Nassers großer Zeit zurücksehnt und manchmal fortschrittliche Einzelaktionen unternimmt, oder die selbstmörderischen und anarchistischen Aktionen einiger von den Massen isolierter, palästinensischer Organisationen (wie z. B. "Schwarzer September"). Doch all diese sporadischen Reaktionen konnten keine Lösung aus der ausweglos scheinenden Situation bringen. Um sein politisches Ansehen nicht zu verlieren, war Sadat, der seit seinem Amtsantritt immer wieder lauthals Vergeltungsaktionen gegen die Israelis angekündigt hatte (obwohl seine ganze Politik in eine andere Richtung lief), gezwungen, eine direkte Lösung des israelisch-arabischen Konflikts anzustreben.

Mit der Rückdeckung Feisals und dem Einverständnis der Sowjetunion, die ebenfalls an einer Lösung des Nahost-Konfliktes interessiert war, aber nicht von der gegenwärtigen schwachen Position aus verhandeln wollte, führten die ägyptischen und syrischen Armeen am 6.10.1973 einen Überraschungsangriff gegen Israel durch. Der militärische Teilerfolg Ägyptens wurde nach dem Waffenstillstand durch die Drohung mit einem Ölboykott der arabischen Ölforderländer unterstrichen. Feisal, einer der reaktionärsten arabischen Politiker, sah hierin eine Chance, seinen bisher geringen politìschen Einfluß in den arabischen Ländern zu verstärken und die Konferenz von Algier zeigte auch den Erfolg der neuen ägyptisch-saudiarabischen Koalition.

\section{Die politischen und wirtschaftlichen Gründe der Erdöldrosselung}

Nach der Konferenz in Kuwait am 16.10.1973 gaben die OAPEC-Mitgliedsländer (Organisation der arabischen ölexportierenden Länder) eine Erdöldrosselung von $5 \%$ bekannt und am 5.11.1973 faßten sie auf einer weiteren Sitzung in Kuwait den Beschluß, die Ölförderung im Verhältnis zur Produktion im September 1973 bis Ende des Jahres um $25 \%$ zu reduzieren. Begründet wurde der Beschluß mit der Forderung an die kapitalistischen Staaten, Druck auf Israel auszuüben, seine Truppen aus den besetzten arabischen Gebieten abzuziehen, damit eine Lösung des Nahost-Konfliktes gewährleistet werden könne. Doch je weiter die Auseinandersetzung führte, um so deutlicher zeigte sich, daß den erdölproduzierenden Ländern im arabischen Raum nicht allein an einem politischen Erfolg gelegen war. Es zeigte sich viel mehr, daß die arabischen Regierungen versuchten, den politischen Anlaß zu einer Drosselung zu nutzen, um gleichzeitig ihre dringenden ökonomischen Probleme zu lösen.

Wir wollen hier einmal auf die wichtigsten Fragen eingehen, die die Motivation für die Öldrosselung abgaben. Am Anfang dieses Artikels haben wir versucht, 
die politische Lage darzustellen, wobei wir feststellen mußten, daß die konservativen Kräfte im arabischen Raum stark an Einfluß gewonnen haben. Sadat und Feisal sind nun die zwei einflußreichsten Politiker in der arabischen Welt. Die ägyptische Bourgeoisie will ihre politische Niederlage wettmachen und sich zwecks besserer ökonomischer Entfaltung (die für sie in der engen Zusammenarbeit mit der Sowjet. union in dieser Weise nicht möglich ist) den kapitalistischen Ländern nähern.

Saudi-Arabien seinerseits ist (wie auch die meisten a ideren arabischen Staaten am Persischen Golf) daran interessiert, mehr politischen Einfluß zu gewinnen, und sucht außerdem nach einer Lösung seiner ökonomischen Schwierigkeiten, die sich um so stärker aufdrängen, je mehr öl aus dem Lande hinausfließt. Sie nehmen nun die Chance wahr, sich den arabischen Massen als kämplerische Vertreter der gesamten arabischen Interessen zu präsentieren und propagieren einen ökonomischen Kampf gegen die westlichen Industriestaaten mit ihrer sog, ,ölwaffe ${ }^{6}$, von deren Wirksamkeit später noch die Rede sein wird.

Wie schon so oft, erleben wir auch heute wieder einmal ein Beispiel für die bewährte Methode arabischer Führungskräfte, rnit großen Reden, kraftvollen Drohungen und theatralischen Gesten der Bevölkerung eine Stärke, Kampfbereitschaft und Einigkeit zu suggerieren, die bis jetzt die Probleme der arabischen Massen nicht zu lösen vermochten und letzten Endes immer wieder in Enttäuschungen mündeten. Die Artikel und Karrikaturen arabischer Zeitungen über die verängstigten Europäer und Amerikaner, die jetzt frieren müssen und weniger Auto fahren können, sind ein Beispiel für die bewußt betriebene falsche Einschätzung der Lage und für die demagogische Aufputschung eines emotionalen Stolzes. Mit dem wachsenden Interesse der arabischen Oberschicht, sich enger an das westliche Wirtschaftssystem anzuschließen, wuchs auch das Interesse an der Lösung des Palästina-Problems, welches einerseits ein Hindernis für die neue Politik sein könnte und andererseits eine ständige Radikalisierungsgefahr für die arabischen Massen mit sich bringt. Deshalb erkannte man auf der Konferenz in Algier zum erstenmal die PLO (Pä̈ästinensische Befreiungsorganisation) als Repräsentant des palästinensischen Volkes an. Ihr Ziel war die Integration des gemäßigten und einflußreichsten Palästinenserfuhrers Arafat.

Doch der politische Anlaß war nicht der Hauptgrund für die beschlossene Herabsetzung der ölförderung. Hinter dem ganzen Konflikt stehen ökonomische Ursachen.

Bei der momentanen Krise der kapitalistischen Weltwirtschaft ist klar, daß die Länder der Dritten Welt, als die schwächsten Glieder des gesamten kapitalistischen Weltsystems, mehr darunter zu leiden haben als die stärkeren. Damit eine katastrophale Ausweitung der Krise in ihren Ländern vermieden wird, miissen die arabischen Regierungen ihre wirtschaftliche Stabilität - die jetzt kopflastig auf dem Rohölexport beruht - festigen. Als Kapitalist versteht Herr Feisal - bisher nur Empfänger der Grundrente - genauso gut wie Herr Rockefeller, wie er sein Kapital am profitabelsten anlegen könnte. Für Saudi-Arabien und die meisten anderen ölexportierenden Länder, wie z. B. Kuwait, Abu Dhabi, Dubai usw, zeigte sich immer deutlicher, daß sie die Produktion ihrer einzigen wertvollen Ware Erdöl gezwungenermaßen überproportional nach dem Bedarf der westlichen Industrieländer steigern mußten, wogegen sie ein Äquivalent erhalten, dessen Wert ständig fällt. Mit der 
Aussicht auf versiegende ölquellen und auf eine zukïnftige Verlagerung des Hauptenergieträgers auf andere, wie z. B. auf die Atomkraft, müssen die Länder größeres Gewicht auf eine vom Rohölexport unabhängigere Wirtschaft legen und den Aufbau - nicht nur der ölverarbeitenden Industrie - sondern auch den anderer Wirtschaftszweige und der gesamten Infrastruktur vorantreiben. Bisher stieß jede Bestrebung dieser Länder nach gezielter Industrialisierung auf den erbitterteWiderstand der Ölgesellschaften und deren kapitalistische Regierungen, die an der alleinigen Beherrschung des Weltmarktes festhalten wollen. So waren diese Länder in ten letzten Jahren zwar reich an Dollars, die in westlichen Geldinstituten gehortet wurden, aber arm an Infrastruktur, Arbeitsplätzen und profitablen Industriezweigen, die ihnen eine eigenständige Kapitalakkumulation ermöglicht hätten. Einige ölexportierende Länder waren wenigstens teilweise in der Lage, diese Probleme zu lösen. Libyen und Algerien setzten ihre ölförderung bewußt herab, und Algerien und der Iran haben bereits eine wenn auch bescheidene Industrie, in die sie einen Teil ihres Kapitals investieren können. Länder wie Saudi-Arabien, Kuwait und der Iran, die sehr stark von den USA abhängig sind, mußten für einen beträchtlichen Teil ihrer ölgewinne riesige Mengen amerikanischer Waffen kaufen. Die USA verkauften z. B. 1973 allein für $3 \mathrm{Mrd}$. Dollar Waffen an die persische Regierung -- ein Handel, dessen Größenordnung selbst für die Vereinigten Staaten einmalig ist. Auf diese und ähnliche Weise fließt ein Großteil des Geldes wieder in die kapitalistischen Industrieländer zurück und in den Förderländern selbst findet keine Kapitalakkumulation statt. Die seit Jahren an die kapitalistischen Staaten und an die mächtigen Ölkonzeme gerichtete Forderung nach einem Aufbau von petrochemischer Industrie und Raffinerieanlagen in ihren Ländern wurde brüsk abgelehnt. Natürlich sind die kapitalistischen Länder nicht daran interessiert, sich für die ökonomische Unabhängigkeit dieser Staaten einzusetzen, da dies einerseits der Verlust von Absatzmärkten zur Folge haben könnte und andererseits sogar eine Gefahr der Konkurrenz in sich birgt.

Dem Drängen der Förderländer versuchten die ôlgesellschaften zögernd damit $\mathrm{zu}$ begegnen, daß sie ihnen Beteiligungen in Industriezweigen anboten, in denen die Kapitalinvestition am größten und die Gewinne am niedrigsten sind. Ein Beispiel hierfür ist die Beteiligung des Iran und einiger arabischer Länder an den Bohrungen in der Nordsee, wo die Investitionen besonders hoch und die zu erwartenden Gewinne relativ begrenzt sind.

Der Widerstand der Ölgesellschaften gegen den Bau eigener Raffinerieanlagen und petrochemischer Industrie in den Förderländern war bisher nicht nur erfolgreich, sondern man bemühte sich auch, die konzerneigenen Raffinerieanlagen, die in den Förderländern bereits vorhanden waren, nach dem Zweiten Weltkrieg allmählich in die Hauptverbraucherländer zu verlegen. Drei wichtige Gründe waren hierfür vorhanden. Einmal die für sie politisch unsichere Lage der Ölländer, zum zweiten der kostspielige Transport von Ersatzteilen (die in den Industriemetropolen hergestellt werden) und zum dritten war der Transport von Fertigprodukten in die Hauptverbraucherländer wesentlich teurer als die einfache Verschiffung des Rohöls. Außerdem sollte auch ein zu starker Devisenabfluß verhindert werden.

Petrochemische Industrie, die der profitabelste Zweig der Ölwirtschaft ist, konnte ebenfalls in den Förderländern nicht angesiedelt werden, da jeder Versuch 
in dieser Richtung an dem starken Widerstand der kapitalistischen Länder scheiterte. Die Petrochemie ist für die Weltwirtschaft so wichtig, daß selbst Länder wie die $B R D$, die weder über eigene Ölquellen noch über eine nennenswerte nationale Ölgesellschaft verfügt, eine umfangreiche Industrie in dieser Richtung aufgebaut hat und daraus riesige Gewinne erzielt.

In einem Interview mit dem Spiegel am 3.12.1973 drückte der saudiarabische Ölminister Jamani auch ganz deutlich den Wunsch seines Landes nach einer Industrialisierung aus. Auf die Frage des Spiegel-Reporters, welchen Beitrag die westlichen Nationen denn leisten könnten, damit Saudi-A rabien soviel öl produziert, wie die ölhungrigen Verbraucherstaaten benötigen, antwortete Herr Jamani: „Das ist sehr einfach. Industrialisieren sie Saudi-Arabien, dann geben wir ihnen soviel öl wie sie brauchen."

Es ist klar, daß Jamani nicht als Vertreter der arabischen Bevölkerung spricht, der die Verbesserung ihrer Lebensbedingungen vorantreiben will, sondem als Vertreter der Großbourgeoisie, die die gleichen Ziele verfolgt wie ihre kapitalistischen Freunde im Westen. Daß die westliche Welt versucht, Jamani (der früher selbst Direktor der ARAMCO war) als einen führenden Vertreter der arabischen Völker darzustellen, ist eine der üblichen Manipulationen, da man genau weiß, daß er und viele seiner Kollegen aus den OPEC-Ländern mehr die Interessen der Ölgesellschaften, als die ihrer arbeitenden Massen wahrnehmen. In den zahlreichen Interviews, die Jamani in der letzten Zeit gegeben hat, erwähnt er kein einziges Mal die Rolle des Ölkartells, obwohl auch ihm bekannt sein dürfte, daß dieses Katell dergefähr. lichste und parasitärste Faktor in seinem Lande ist.

Der Druck, den die Förderländer mit ihrem Ölboykott nun ausüben, ist zwar nichi sehr stark (eine Dollarspekulation dieser Länder hätte vor allem die USA in eine viel größere Verlegenheit bringen können, und inwieweit sich die beschlossene Öldrosselung tatsächlich realisieren ließ, ist immer noch nicht klar), dennoch hat er die Auseinandersetzung um die Industrialisierungsfrage wieder in Gang gesetzt. Nach Meldungen der jüngsten Zeitungsberichte zu urteilen, werden sich in Zukunft die Industrieländer - vor allem einige EG-Mitglieder - stärker für die Projekte in den arabischen Ländern interessieren.

Die Problematik der Listenpreise und die Rolle der Mineralölgesellschaften bei den jüngsten Auseinandersetzungen

Da über den Preis, den die ölexportierenden Länder für ihr Rohöl bekommen, große Verwirrung herrscht, wäre es besonders jetzt - wo wir fast täglich über neue Preiserhöhungen in der Mineralölbranche lesen können - notwendig, etwas über die Preisentwicklung und die Beziehungen der Förderländer zu den ölkonzernen und den kapitalistischen Staaten zu sagen.

Wir wollen hier nicht die gesamte historische Entwicklung behandeln, doch wir wollen hier einmal ein Beispiel dafür anführen, wie ungleichmäßig die Profitverteilung in der Erdölbranche ist. Im Jahre 1947 überprüfte eine amerikanische Senatskommission die Preise für das Rohöl, welches die US-Marine während des II. Welt- 
krieges von der ARAMCO (die Arabian American Oil Company war 100prozentig im Besitz der vier größien amerikanischen Ölgesellschaften) bezogen hatte. Die Kommission kam zu dem Ergebnis, daß im Jahre 1945 die US-Marine für einen Barrel (159 Liter oder 1 Faß) Rohöl 1,05 Dollar an die ARAMCO zahlte. Von diesem Preis erhielt die saudiarabische Regierung 21 Cents und die Scheichs von Kuwait 15 Cents pro Barrel, das restliche Geld floß der ARAMCO als Gewinn in die Tasche, da sie bei diesem Geschäft weder Verarbeitungs- noch Verteilerkosten hatte und obwohl ihr auch keine Belastung durch den Transport entstand, da das Rohöl am Persischen Golf - wo man es förderte - durch die Schiffe der US-Marine selbst verladen wurde. An dieser ungleichen Gewinnverteilung hat sich bis jetzt wenig geändert.

Das erste Land, welches gegen diese Ausplünderung den Kampf aufgenommen hatte, war der Iran. Dort wurde 1951 unter der Führung des damaligen Ministerpräsidenten Mossadegh die gesamte Erdölindustrie verstaatlicht. Mossadegh legte dem Internationalen Gerichtshof in Den Haag Dokumente vor, in denen die Ausbeutung durch die Konzerne so krass zum Ausdruck kam, daß ihm das Gericht in seinen Forderungen Recht geben mußte. Der CIA stürzte 1953 die Mossadegh-Regierung und statuierte damit ein Exempel für all die Länder, die evtl. ähnliche Wege beschreiten wollten. Der Putsch schuf gleichzeitig für die größten amerikanischen Ölgesellschaften die Möglichkeit, mit dem größten Anteil an der iranischen Ölförderung in das persische Ölgeschäft einzusteigen, welches früher allein von den Engländern beherrscht wurde.

Als sich Ende der 50er Jahre die Ölkonzerne einen erbitterten Preiskampf lieferten, unter dem die ölexportierenden Länder am meisten zu leiden hatten, wurde unter der Initiative der damaligen irakischen Regierung die OPEC (Organisation der ölexportierenden Länder) gegründet in der Hoffnung, gemeinsam auf die Preisbildung einen gewissen Einfluß nehmen zu können. In den ersten Jahren ihres Bestehens konnte die OPEC wegen der geringen Zahl ihrer Mitglieder, ihrer inneren Widersprüche und der starken Abhängigkeit der einzelnen Mitgliedstaaten von den Ölgesellschaften keinen nennenswerten Einfluß ausïben. In der Anfangsphase lehnten es die Ölgesellschaften sogar ab, mit dieser Organisation zu verhandeln. Erst Ende der 60er Jahre kam es allmählich zu ernsthaften Verhandlungen. Es gab folgende Gründe für diese Wende:

1. Mittlerweile hatten sich alle wichtigen ölexportierenden Länder (die Mitgliederzahl stieg von 4 auf 11 an) der OPEC angeschlossen und verliehen der Organisation mehr Gewicht.

2. Die ernsthaften Forderungen Libyens und Algeriens und deren Erfolge ebneten den anderen Mitgliedstaaten den Weg, selbst auch höhere Forderungen zu stellen. Allerdings waren die Forderungen Libyens und Algeriens auch mit dem antiimperialistischen Kampf verknüpft, wogegen die übrigen Länder nur ein paar Cents mehr für ihr o̊l verlangten und das auch nur unter dem Druck der öffentlichen Meinung in ihren eigenen Ländern.

3. Das Heranwachsen von hauptsächlich italienischen, französischen und japanischen Ölgesellschaften, die vom ölkartell unabhängig waren, bildete allmählich eine starke Konkurrenz für die „Großen Sieben“ auf dem interna- 
tionalen O̊lmarkt und veranlaßte sie, den OPEC-Ländern bessere Verträge anzubieten.

4. Mit der allgemeinen Entwicklung des politischen Bewußtseins in der Dritten Welt - insbesondere in den arabischen Ländern - mußten sich auch die reaktionärsten Regierungen stärker für die Rechte ihrer Länder engagieren.

Die Konferenzen von Caracas, Teheran und Tripolis waren die verschiedenen Etappen einer langen Auseinandersetzung, die Ende der 60er Jahre begann und bis 1971 andauerte.

Eines der wichtigsten Ergebnisse der Konferenz in Caracas war die am 12. 12. 1970 verabschiedete Resolution Nr. 120, die u. a. besagte, daß .der Listenpreis aller OPEC.Mitgliedstaaten immer an den Preis angeglichen werden muß, der von einem OPEC-Land in Einzelverhandlungen mit den Oólgesellschaften erzielt worden ist.

Da zu diesem Zeitpunkt Libyen und Algerien bereits mit den einzelnen Ölkonzernen in Verhandlungen standen und abzusehen war, daß diese beiden OPECLänder sowohl wegen ihrer bevorzugten geographischen Lage am Mittelmeer, als auch wegen ihrer konsequenteren Haltung relativ günstige Preise erzielen würden, wäre es eigentlich vernünftig gewesen, wenn die übrigen Mitgliedstaaten das Resultat dieser Verhandlungen abgewartet hätten, bevor sie sich zu neuen Preisverhandlungen trafen. Es muß also als großer Erfolg für die Ölgesellschaften gewertet werden, daß sich die Länder vom Persischen Golf bereits vor dem Abschluß der libysch-algerischen Verhandlungen in Teheran trafen. In dieser Konferenz wurden zum erstenmal gemeinsam die Rohölpreise zwischen den ölexportierenden Ländern am Persischen Golf und den Ölgesellschaften für die nächsten fünf Jahre festgesetzt. Der Listenpreis wurde auf etwa 2,25 Dollar pro Barrel (dies ist nur ein Durchschnittswert, da sich die Preise im einzelnen nach der unterschiedlichen Qualität des Ools richten) festgesetzt, wovon den Förderländern etwa 1,30 Dollar pro Barrel Rohöl als fiskalische Abgaben ausgezahlt werden sollten. Außerdem kalkulierte man eine 2,5\%ige jährliche Inflationsrate ein, obwohl die Geldentwertung des Dollars bereits damals über $3,5 \%$ lag. Eine zusätzliche Liefergarantie sicherte den ölkonzernen eine unumschränkte Menge Rohöl, welches sie je nach Bedarf fördern konnten. Damit hatten die Konzerne der schon damals drohenden öldrosselung mancher Länder vorgebeugt.

Gemessen an der damaligen politischen und wirtschaftlichen Weltlage, hatte das Ölkartell mit Hilfe der Regierungen am Persischen Golf - die eng mit den Konzernen zusammenarbeiten - erfolgreiche Verträge abschließen können. Für die paar Cents, die die OPEC-Länder jetzt mehr erhielten, veranstaltete damals die westliche Presse eine solche Hetzkampagne, daß man den Eindruck gewinnen mußte, als sei all das, was die kapitalistische Welt im Laufe ihrer Geschichte den anderen Völkern geraubt hatte, jetzt mit einem Schlag durch die ölproduzierenden Länder zurückgeholt worden. A ber hält man sich einmal die Problematik der Preisbildung beim Rohöl vor Augen und analysiert die tatsächliche Gewinnverteilung, so wird offensichtlich, wie schlecht die Förderländer ihre oft einzigen nationalen Bodenschätze verkaufen.

Die Preispolitik der O̊lkonzerne ist so raffiniert angelegt, daß selbst Experten 
nicht in der Lage sind, ihre tatsächlichen Gewinne zu ermitteln. Ihre Taktik besteht darin, niemals den effektiven Preis anzugeben, den sie für das Rohöl zahlen, sondern sie operieren mit verschiedenen Preisen, die sich nach verschiedenen Kriterien aufschlüsseln lassen. Bei Preisverhandlungen mit den OPEC-Ländern wird z. B. immer yon einem Listenpreis gesprochen, uber den auch die Presse ausfuhrlich berichtet und der das zu sein scheint, was diese Länder für ihr Rohöl bezahlt bekommen. In Wirklichkeit liegt der Betrag, den die Förderiänder erhalten, sehr viel niedriger. Der Listenpreis (auch posted price oder fiktiver Preis genannt) ist aber lediglich die Basis, auf der die fiskalischen $A$ bgaben an die Förderländer errechnet werden.

Der Listenpreis regelt aber nicht nur den Anteil, den die Förderländer erhalten, sondern er bestimmt auch den Gewinnanteil der Fördergesellschaften am Rohol. Die Berechnung der Gewinnanteile wird so gehandhabt, daß man vom Listenpreis die Förderkosten (die am Persischen Golf bei 10-12 Cents pro Barrel liegen) und die Förderzinsen (die auch Royalties genannt werden und immer $12,5 \%$ des jeweiligen Listenpreises ausmachen) für die Ölländer abzieht und die verbleibende Summe im Verhältnis 55 : 45 zwischen den Förderländern und den Ölgesellschaften aufteilt. Dabei wird der Gewinnanteil der Förderländer als Steuerabgabe deklariert, was für die Konzerne den Vorteil hat, daß diese als Auslandssteuern bei der Gewinnermittlung in ihren Heimatländern abgesetzt werden können. Wird der Listenpreis heraufgesetzt, so erhöhen sich also nicht nur die fiskalischen Abgaben an die Förderländer, sondern auch der Gewinnanteil der Ölgesellschaften steigt automatisch prozentual mit an. Auf diese Tatsache wird in der Presse nie deutlich genug hingewiesen, so daß der Eindruck entsteht, als brächte die Erhöhung der Listenpreise nur finanzielle Vorteile für die OPEC-Länder.

Um diese Verrechnungsart einmal zu veranschaulichen, sei hier die halbamtliche Teheraner Zeitung Etielaat vom 22. 12. 1973 zitiert, die den iranischen Rohölpreis, der in der Teheraner Konferenz 1971 ausgehandelt wurde, folgendermaßen aufschlüsselt:

Listenpreis pro Barrel Rohöl

227,4 US-Cents

Davon entfielen auf die:

Förderländer für fiskalische Abgaben

Förderkosten

Ölgesellschaften als Provision

Ölgesellschaften als Rabatt

\section{2,3 US-Cents \\ 12,0 US-Cents \\ $25-35,0$ US-Cents \\ $48,1-58,1$ US-Cents}

Diese Aufstellung erwähnt nicht den Royalty, aber er muß in diesem Fall in den fiskalischen Abgaben an den Iran enthalten sein. Es entfielen also ganze 1,32 Dollar pro Barrel Rohöl auf das Forderland.

Doch die ölländern schneiden nicht nur im Vergleich mit den o̊lgesellschaften (die neben dem 45prozentigen Gewinn aus dem Listenpreis ja auch noch die Einnahmen aus Transport, Verarbeitung und Verteilung an jedem Barrel Öl einstecken) schlecht $a b$. Die kapitalistischen Regierungen erhalten ebenfalls durch hohe Mineralölsteuern wesentlich mehr Einnahmen aus dem Öl als die Förderländer 
selbst. Die Frankfurter Allgemeine Zeitung vom 20.10.1973 schreibt dazu: „1971 beispielsweise ... sind in der BRD für alle Produkte, die aus einer Tonne Rohöl gewonnen werden konnten, etwa 260,00 DM erlöst worden. Genau die Hälfte dieses Erlöses (nämlich 130,00 DM) hat an das Finanzamt abgeführt werden müssen, und zwar 104,00 DM Mineralölsteuer und 26,00 DM MWSt. . . Dabei haben die effektiven Aufwendungen für die Förderung je Tonne Rohöl etwa $8 \mathrm{DM}$ betragen, für fiskalische Abgaben an die ölländer etwa 40,00 DM ..."

Demnach erhielt allein die Bundesregierung mehr als das Dreifache des Betrages, den die Förderländer für ihr öl erzielen konnten. Hinzu kommt noch, daß sich seit der Teheraner Konferenz die Verhältnisse für die OPEC-Länder immer ungünstiger entwickelt haben. Einmal stieg die Inflationsrate auf 6-8\% an und minderte stark das Realeinkommen der Förderländer und zum anderen hatten sich die Ölpreise auf dem Weltmarkt fast verdoppelt.

In der Konferenz von Wien am 8.10.1973 wollten die OPEC-Länder diese negative Entwicklung mit den Ölgesellschaften besprechen und zu neuen Preisvereinbarungen kommen. Am 6. 10. 1973 war aber überraschend der Krieg im Nahen Osten ausgebrochen und die Ölgesellschaften nahmen dies zum Anlaß, um die Verhandlungen ergebnislos zu vertagen.

Den willkommenen Aufschub benutzten die Ölkonzerne geschickt, um sofort eine groß angelegte Kampagne gegen die ölexportierenden Länder zu starten und um die Basis für erhebliche Preissteigerungen vorzubereiten.

Obwohl zu dieser Zeit von der OPEC aus weder von neuen Preisen noch von einer Öldrosselung die Rede war, war die gesamte westliche Presse voll von Spekulationen, die sich teilweise schon wie fertige Tatsachen lasen. Durch die Meldungen über Ölknappheit, geschlossene Tankstellen, Hamsterkäufer und Preissteigerungen wurde die Stimmung angeheizt. Überall war zu hören und zu lesen, daß das öl knapp geworden sei, obwohl rätselhaft blieb, wo die Ursache für diese Verknappung liegen konnte.

Die einzige, die im Oktober tatsächlich ihre Rohölförderung drosselte, war die Ölgesellschaf $t$ ARAMCO, die die gesamte Ölproduktion in Saudi-Arabien kontrolliert und die, wegen einer evtl. Beschädigung ihrer Pipelines durch den Krieg, die Tagesproduktion um 250000 Barrel kürzte.

Die Vorgänge Ende des Jahres 1973 sind typisch für die Art und Weise, wie Monopole in der Lage sind, die öffentliche Meinung zu ihren Gunsten zu mobilisieren. Ein sehr großer Teil der Massenmedien hilft ihnen dabei. Auch die kapitalistischen Regierungen haben kein Interesse, gegen das Ölkartell vorzugehen, da sie einerseits selbst an den Preiserhöhungen profitieren und andererseits politisch dem Kartell gegenüber zu schwach sind.

Damit man versteht, wie das Kartell den Nahost-Konflikt ausgenutzt hat, muß man die wirtschaftliche Lage der Konzerne in den letzten Jahren berücksichtigen. In den letzten Jahren konnten die großen Ölgesellschaften ihre Gewinne nicht so steigern, wie sie es sich vorstellten und sie konnten nicht genug Kapital akkumulieren, um ihre Monopolstellung nach ihren Wünschen weiter auszubauen. Zu diesem Thema schreibt der Stern vom 26.11.1973: ,Noch vor dem Embargo schätzte die Chase Manhattan Bank, daß die US-Industrie weltweit eine Billion Dollar für In- 
vestitionen und laufende Operationen brauche, um die wachsende Energienachfrage decken zu können. Um genügend Geld für diese Investments zu bekommen, müssen die Ölgesellschaften - so empfahl die Bank - ihre Gewinnspanne verdoppeln. Chase Manhat tan Bank-Vorsitzender ist David Rockefeller."

Die Ölkonzerne hatten sich längst nach dieser Prognose gerichtet. Ende des Jahres 1972 verknappten sie in den USA die ölversorgung planmäßig. Sie steigerten damit die Nachfrage, konnten Preiserhöhungen rechtfertigen und was das wichtigste war, sie brachten die meisten freien Tankstellen an den Rand des Ruins. Die freien Tankstellen und Heizöllieferanten, die von den Lieferungen der Monpolgesellschaften abhängig sind, bekamen nicht mehr genug Benzin und Heizöl, um ihr Geschäft aufrechterhalien zu können. (Genau dasselbe praktizieren die großen Konzerne heute auch in der BRD und in anderen europäischen Ländern.) Sie mußten einen großen Teil ihres Bedarfs aus ausländischen - hauptsächlich aus Kanada kommenden - Importen decken. Dieses auf dem freien Weltmarkt ohnehin schon teuere o̊l war durch Nixon (der, wie jeder US-Präsident im Interesse der Ölkonzerne handeln muß) mit hohen Einfuhrzöllen belegt worden, was katastrophale Auswirkungen auf die kleinen Unternehmen hatte, die am monopolisierten Versorgungsnetz der Großen nicht angeschlossen waren. Die Strategie war erfolgreich. Der Gewinn stieg bei den fünf größten US-Ölfirmen im dritten Quartal 1973 um (in Klammer der durchschnittliche Jahresgewinnanstieg 1970-73):

Exxon

$80,7 \%(22,8 \%)$

Texaco

$48,2 \%(15,8 \%)$

Mobil Oil

Standard Oil of California

$64,1 \%(17,9 \%)$

Gulf Oil

$50,7 \%(19,6 \%)$

$90,9 \%(10,8 \%)$

Diese überproportionalen Gewinnsteigerungen sollten nun durch die gunstige Gelegenheit, die der Nahost-Krieg bot, nochmals aufgestockt werden. Daß dies möglich war, zeigte sich auch bald an den enormen Preiserhöhungen - besonders auf dem Heizölsektor.

Nach der Wiener Konferenz, die keine neuen Vereinbarungen zwischen den Ölgesellschaften und den OPEC-Ländern brachte, trafen sich die OAPEC-Mitglied. staaten (Organisation der arabischen ölexportierenden Länder) am 16.10.1973 auf einer Konferenz in Kuwait, um über die neu entstandene Situation zu beraten. Die jüngsten Preiserhöhungen der Endprodukte auf dem Mineralölmarkt bestärkten die arabischen Förderländer in der Absicht, die Rohölpreise von ihrer Seite aus zu erhöhen.

Am 17. 10. 1973 wurde bekanntgegeben, daß sich die OAPEC-Mitglieder für eine Anhebung des Listenpreises um $70 \%-d$. h. von ca. 3 auf 5 Dollar pro Barrel - und für eine Anhebung des Marktpreises (das ist der Preis für den Teil des Öls, den die Förderländer aus eigenem Anteil produzieren und dem sie größtenteils an die Ölkonzerne weiterverkaufen, deshalb nennt man ihn auch Rückkaufpre is) um $17 \%$ - d. h. von 3,05 auf 3,65 Dollar pro Barrel - aussprachen. Außerdem beschloß man eine 5 prozentige ôldrosselung. 
Die Ankündigung einer Öldrosselung bestärkte die Ölgesellschaften in ihrer Taktik, eine künstliche ölverknappung voranzutreiben, obwohl sich zu diesem Zeitpunkt das Ölembargo wegen der langen Tankerwege (die Tanker brauchen ca. 4-6 Wochen, um vom Persischen Golf zu den europäischen Bestimmungshäfen zu gelangen) noch nicht auswirken konnte. Gleichzeitig stiegen die Tankerfrachtraten bis zu $800 \%$ an (ein Großteil der Tanker ist im Besitz der Ölgesellschaften), was ein sicheres Zeichen für ihre volle Ausiastung ist. Auf den geäußerten Verdacht hin, daß viele Tanker zwischen Schottland und Island kreuzten, um dorthin dirigiert werden zu können, wo für das öl die höchsten Preise zu erzielen seien, antwortete ein Vertreter der Ölgesellschaften, daß diese Tanker nur deshalb nicht in Rotterdam löschten, weil die Vorratstanks alle voll seien. Ungewollt widersprach er damit den Gerüchten ïber eine ölknappheit. Doch nicht nur, daß das o̊ll keineswegs knapp war, es zeigte sich auch, daßs die von der Bundesregierung vorgeschriebenen Mindestreserven der Mineralölgesellschaften yon 6,9 Mill. Tonnen im November auf 10,7 Mill. Tonnen aufgestockt werden konnten. Die Ölvorräte der Bundesregierung stiegen im gleichen Zeitraum von 7,5 Mill. Tonnen auf 9 Mill. Tonnen an.

Am 5.11.1973 kündigten die OAPEC-Länder eine weitere Verschärfung des Ölboykotts an. Die Ölförderung sollte bis Ende des Jahres um $25 \%$ gekürzt werden. An dieser Stelle möchten wir nochmals auf die Problematik der Öldrosselung eingehen. Am Anfang dieses Artikels haben wir davon gesprochen, daß die arabischen Länder aus politischen und vor allem wirtschaftlichen Erwägungen heraus ein Interesse daran hatten, mit einem ölboykott ihren Vorstellungen mehr Gewicht zu verleihen. Tatsächlich sind sie auch in dieser ganzen Auseinandersetzung ihren Zielen näher gekommen. Aber inwieweit dies die direkte Folge einer tatsächlich gedrosselten ölförderung war, läßt sich nicht abschätzen. Die praktische Handhabe der Ölboykottierung birgt in sich einige widersprüchliche Faktoren. Einmal beschlossen die OAPEC-Länder eine allgemeine Drosselung der Erdölproduktion um bis zu $25 \%$, die sich - wenn man die Verbraucherländer, die dieses öl konsumieren, in Betracht zieht - auf die einzelnen vom arabischen O̊l abhängigen Industriestaaten prozentual hätte auswirken müssen. Andererseits haben die arabischen Länder aber die Industrieländer in ,freundliche ${ }^{66}$ und ,feindliche "Nationen aufgeteilt und nur einen Boykott gegen die sog. "feindlichen", d. h. konkret gegen die USA und die Niederlande ausgesprochen.

Berücksichtigt man aber die Praxis der internationalen ölwirtschaft, so kann man sich allerdings kaum vorstellen, wie sich dieser ölboykott hätte realisieren lassen können. Zwar wird das öl in den genannten Ländern gefördert, aber wenn man erstens die Macht der Ölkonzerne in diesen Ländern kennt, kann man kaum annehmen, daß es den arabischen Regierungen ohne weiteres möglich sein sollte, den Ölhahn in den konzerneigenen Förderanlagen abzudrehen. Sie könnten zwar ihren eigenen Förderanteil reduzieren, jedoch ist dieser zu gering in den meisten Ländern, um größere Boykottmaßnahmen wirksam werden zu lassen. Außerdem ist das gesamte Verteilungssystem nicht in Händen der Araber, sondern in den Händen der Ölkonzerne konzentriert, die das Rohöl nach den Bedürfnissen des Marktes verteilen und auf diese Belieferung haben die arabischen Staaten keinerlei Einfluß. Nur die ôlkonzerne entscheiden und wissen, wo welches öl ausgeliefert wird. 
Wir können hier nicht sagen, ob sich das ölembargo tatsächlich in irgendeinem Land durch spürbar verringerte ölimporte (die durch die arabischen Länder verursacht wurden) bemerkbar gemacht hat. Darüber liegen weder Zahlen noch Berichte vor. Doch wir können sagen, daß sich der Boykott von Seiten der Mineralölkonzerne in der Weise auswirkte, daß eine strategische Ölverknappung betrieben wurde. Für die letzte Behauptung gibt es zahlreiche Dokumente, die diese Vermutungen bestärken. So schreibt z. B. die Zeit vom 30.11.1973: ,N Nach dem Beschluß der arabischen Länder ... eine Drosselung der ölförderung um $25 \%$. . ist in diesen Ländern bisher jeder Tanker beladen worden. Die Mineralölgesellschaften haben jedoch weniger Tanker in diese Region beordert ... In welchem Ausmaß die arabischen Länder bislang die Förderung gedrosselt haben, ist unklar, da die Fördergesellschaften jede Auskunft verweigern."

Die britische Wochenzeitschrift The Economist berichtete sogar, daß den Schiffahrtsberichten der Londoner Versicherungsbörse Lloyds zufolge die Verschiffung an den arabischen Häfen Ende November, Anfang Dezember um rund $40 \%$ uiber dem Vorjahresniveau lag. Dennoch wurde die Produktion in den Rotterdamer Raffinerien Anfang Dezember um 25 bis $35 \%$ gekürt.

Am 23, 12. 1973 trafen sich die ölproduzierenden Länder am Persischen Golf in Teheran und beschlossen, den Listenpreis auf 11,65 Dollar (wovon die Förderländer anteilmäßig rund 7 Dollar erhalten würden) pro Barrel anzuheben. Die neuen Preise sollten ab 1.1.1974 in Kraft treten.

Die Überlegungen, die wir bei dieser Preiserhöhung anstellen müssen, sind erstens, ob die Ölgesellschaften diese Preise akzeptieren werden (Meldungen über eine Stellungnahme der betroffenen ölkonzerne lagen bis jetzt (Mitte Januar) noch nicht vor), zweitens ob diese Preise bis jetzt tatsächlich realisiert worden sind und drittens, was mit den langfristigen Verträgen geschieht, die alle diese Länder mît den ölgesellschaften abgeschlossen haben, durch die sie an sich verpflichtet sind, bis 1975 ihr Rohöl zu den 1971 in Teheran festgesetzten Preisen zu verkaufen. All diese entscheidenden $F$ ragen wurden bis jetzt in der Presse nicht behandelt.

Die Beschlïsse von Kuwait (16.10.1973) und Teheran (23.12.1973), die Erdölpreise in Zukunft ohne vorherige Verhandlungen mit den Ölkonzernen festzusetzen, können aber nur dann richtig beurteilt werden, wenn man den Charakter dieser Regierungen genau kennt. Gerade die Regierungen am Persischen Golf sind bekanntermaßen das direkte Produkt des ölkartells und der englischen und amerikanischen Politik, die in brutalster Weise gegen den Widerstand der antiimperialistischen Massen ihrer eigenen Länder vorgehen.

Es wäre verfehlt, diese Beschlüsse als antiimperialistische Schritte zu bezeichnen. Daß die Preise des Rohöls früher oder später erhöht werden mußten, war unvermeidlich. Aber inwieweit diese Preiserhöhungen im Widerspruch zu den Interessen der großen Ölkonzerne stehen, kann man mit dem jetzigen Informationsstand nicht genau sagen. Wir müssen die neuen Verträge, die zwischen den O̊lförder * ländern und den Ölgesellschaften abgeschlossen werden, abwarten. Nur dann kann man eine genaue Analyse darüber machen.

Am Ende dieser Ausführungen soll hier noch ein besonders augenfälliges Beispiel für die Manipulationen um die Ölpreise angeführt werden. Anfang Dezember 
1973 meldeten die Tageszeitung auf der ersten Seite, der Iran habe seinen Rohölprois auf 17,36 Dollar pro Barrel heraufgesetzt. Diese Meldung wurde mit keinem Wort über die Umstände, unter denen dieser Preis zustande gekommen ist, erläutert. Natürlich bringt der Konsument solchermaßen informiert leichter Verständnis für die Preiserhöhungen der Mineralölkonzerne auf. Keiner der Kommentatoren und Wirtschaf sexperten stelite aber die kritische Frage, warum ausgerechnet die persische Regierung, die so eng mit den ölkonzernen zusammenarbeitet und als Preisbremse in der OPEC bekannt ist, plötzlich einen so unwahrscheinlichen Schritt unternehmen sollte. In diesem Fall handelt es sich aber nicht um eine generelle Preiserhöhung, die die persische Regierung auch mit bestem Willen ohne die $\mathbb{Z} u s t i m$ mung der ölkonzerne nicht hätte durchsetzen können, sondern lediglich um einen bei einer Rohölversteigerung erzielten Höchstpreis. Die größte iranische Tageszeitung $R$ ayhan kommentierte dieses Ereignis am 12.12. 1973 folgendermaßen:

,Das persische Rohöl wurde bei einer Versteigerung zu 17,36 Dollar pro Barrel verkauft ... Zur Zeit wird das persische Rohöl zu 3,3 Dollar pro Barrel an das Konsortium (das sind die sieben großen Ölgesellschaften, die auch nach der formalen Verstaatlichung dennoch die iranische Ölwirtschaft kontrollieren - Anm. d. Verf.) verkauft ... Die Summe, die am Dienstag versteigert wurde, ist die Hälfte des Teils, den der Iran aus eigenem Förderanteil für das Jahr 1974 zur Verfügung hat. Der Iran produziert zur Zeit 6 Mill. Barrel Rohöl pro Tag. Aber von dieser Summe kann der Iran nur 450000 Barrel täglich eigenständig verkaufen. Alles andere wird an das damalige Konsortium, das aus englischen, amerikanischen und holländischen Ölge sellschaften bestanden hat, abgegeben. Das Konsortium hat te bis März dieses Jahres die iranische ölindustrie kontrolliert. Danach schlossen die Ölgesellschaften mit der NIOC (Nationale Iranische Ölgesellschaft - Anm. d. Verf.) einen 20jährigen Liefervertrag. ... Die Namen der Firmen, die das Öl ersteigerten, wurden nicht bekanntgegeben."

Im gleichen Artikel schreibt die Kayhan daß der Preis von 17,36 Dollar pro Barrel ein Spitzenpreis war, der für besonders hochgradiges Öl erzielt wurde. Die anderen Ölqualitäten wurden zu einem Durchschnittspreis von 16 Dollar verkauft. Leider wurden die Mengenverhältnisse nicht angegeben.

Die gesamte versteigerte Menge betrug also nur 237500 Barrel, was nicht einmal $4 \%$ der iranischen Tagesproduktion entspricht. Wieviel wirklich zu diesem Höchstpreis versteigert worden ist und mit welchen Lieferbedingungen der Handel zustande kam, ist bis jetzt noch nicht geklärt.

Auch in diesem Fall scheint die Wirkung der Schlagzeilen größer zu sein als die Auswirkungen, die diese Preiserhöhung selbst hat. Dieses konkrete Beispiel zwingt uns, gegenüber jeder neuen Preismeldung, die in den kapitalistischen Ländern bekannt wird, skeptisch zu sein. Man hört fast täglich von neuen Preissteigerungen auf dem Rohölmarkt, ohne daß man ein einziges Mal darüber informiert wird, wann und mit wem diese Preiserhöhungen realisiert worden sind.

In dem jüngsten Nahost-Krieg erzielten die Araber zwar am Anfang einige militärische Erfolge, die man im Hinblick auf die aggressive Haltung Israels positiv einschätzen muß. Doch der weitere Verlauf des Konfliktes brachte bis jetzt für die revolutionären Bewegungen im Nahen Osten nur negative Ergebnisse. Zum Schluß 
wollen wir noch einmal diese negativen Ergebnisse zusammenfassen:

- Sadat festigte seine Position im In- und Ausland. Die bevorstehende Lösung des arabisch-israelischen Konfliktes muß im Zusammenhang mit den Bestrebungen der arrivierten ägyptischen Großbourgeoisie gesehen werden, sich an den westlichen Kapitalmarkt anzuschließen. Obwohl offensichtlich ist, daß die USA Israel auch weiterhin voll und ganz unterstützen werden, beschloß Sadat beim ersten Besuch Kissingers in Kairo, die diplomatischen Beziehungen zu den USA wieder aufzunehmen. Den Berichten der Time zufolge wird eine amerikanische Firma demnächst mit dem Bau einer Pipeline von der Stadt Suez zum Mittelmeer beginnen. Dieses 365 Millionen-Dollar-Projekt ist seit langer Zeit die erste große amerikanische Kapitalinvestition in $\AA$ gypten, aber sicher nicht die letzte.

Die reaktionären Kräfte der arabischen Länder gewannen in einem solchen $\mathrm{Maß}$ an Einflußs, wie man es sich bei der wachsenden antiimperialistischen Atmosphäre niemals vorstellen konnte. Der Konflikt, den Länder wie Saudi: Arabien und Kuwait mit den kapitalistischen Staaten unter Zuhilfenahme der sog. „ölwaffe" austragen, sollte von uns keinesfalls als Unterstützung der revolutionären Bewegungen eingeschätzt werden. Als Kapitalisten nutzten sie die Gelegenheit aus, zum einem ihre überproportionale Erdölproduktion einzuschränken und zum anderen durch direkte Verhandlungen mit einigen EGLändern den Kapitalisierungsprozeß ihrer Länder zu beschleunigen, um dadurch in Zukunft bessere Verwertungsmöglichkeiten für ihr Kapital zu schaffen. Natürlich ist eine Industrialisierung dieser Länder nicht negativ zu bewerten, doch diese neuen Kapitalverflechtungen verstärken auch wieder ihre Abhängigkeit von den westlichen Industrienationen.

- Die USA, deren Ölkonzerne den größten Teil der nahöstlichen Ölreserven kontrollieren, sehen in der Lösung des Nahost-Konfliktes die Beseitigung eines Krisenherdes, der ihre riesigen Profite aus diesem Teil der Welt ernsthaft in Gefahr bringen konnte. Die ständigen Reisen Kissingers in den Nahen Osten müssen in dem Rahmen gesehen werden, daß sie sowohl die eigene als auch die israelische Position festigen, um in Zukunft stärker gegen die revolutionären Bewegungen der Palästinenser, Dhofars, Süd-Jemens und anderer evtll. neu entstehender Bewegungen vorgehen zu können. $\mathbb{Z u}$ erwähnen ist außerdem, daß die durch den Nahost-Konflikt ausgeloste „,Energiekrise ${ }^{66} \mathrm{er}$ heblich zur Stabilisierung des Dollars beigetragen hat.

Durch die neue Entwicklung wërden die arabischen Länder stärker an das kapitalistische Weltwirtschaftssystem angeschlossen als je zuvor. 